\title{
Latent Class Analysis of Brain Injury Symptomatology among College Students
}

\author{
Karen Hux, $\mathrm{PhD}^{1}$ Jessica Brown, $\mathrm{PhD}^{2}$ Matthew Lambert, $\mathrm{PhD}^{1}$ \\ ${ }^{1}$ Department of Special Education and Communication Disorders, \\ University of Nebraska-Lincoln, Lincoln, Nebraska \\ 2 Department of Speech-Language-Hearing Sciences, University of \\ Minnesota, Minneapolis, Minnesota \\ Address for correspondence Karen Hux, PhD, Department of \\ Special Education and Communication Disorders, University \\ of Nebraska-Lincoln, 351 Barkley Memorial Center, Lincoln, \\ NE, 68583 (e-mail: khux1@unl.edu).
}

Curr Res Concussion 2017;4:e14-e22.

\begin{abstract}
Keywords

- mild brain injury

- concussion

- recovery

- sequelae

- post-secondary education

- general health
\end{abstract}

Background Incidents potentially causing mild brain injury (BI) are common, and most people recover rapidly; however, a subset experiences long-lasting challenges. Objective This study used latent class analysis to identify a subset of college students presenting chronic symptomatology consistent with a mild BI diagnosis and pseudoclass mean equality tests to examine relations between latent classes and $\mathrm{BI}$ event and academic outcome variables.

Methods Participants were 118/423 undergraduates self-reporting possible mild Bls through a survey about general health, daily habits, academic performance, and potential BI events. Twenty-four cognitive, physiological, or socio-emotional sequelae served to identify symptomatology profiles.

Results A three-class model including 11\% with high symptomatology, $49 \%$ with moderate symptomatology, and $40 \%$ with negligible symptomatology provided excellent fit and entropy. Symptoms best separating high and moderate classes were memory, thinking speed, new learning, and attention problems. Mean equality tests revealed no significant difference in number of Bl events across classes, but high symptomatology respondents were significantly less likely to lose consciousness and significantly more likely to have lower grade point averages and to have failed courses than moderate symptomatology respondents.

Discussion Cognitive problems are paramount in distinguishing college students with chronic high symptomatology following $\mathrm{BI}$ from those with moderate and negligible symptomatology. Because high symptomatology class individuals differ academically from their counterparts, a functional consequence of mild BI appears to exist.

Conclusion About 1 in 10 undergraduate students self-reporting $\mathrm{BI}$ events experiences chronic symptomatology affecting general health and academic achievement. Because they may benefit from supportive services, accurate identification is critical.

\section{Latent Class Analysis of Brain Injury Symptomatology among College Students}

Increased awareness among professionals and the lay public about potential short- and long-term consequences associated with mild brain injuries (BIs) has prompted consider-

received

September 23, 2016 accepted after revision August 4, 2017
DOI https://doi.org/

$10.1055 / \mathrm{s}-0037-1606578$. ISSN 2368-0539. able interest in the topic of identification and recovery patterns. One issue that arises repeatedly relates to distinguishing injuries resulting in chronic symptoms from those to which people appear to have relatively rapid and uncomplicated recoveries. Specifying and identifying the enduring cognitive, physiological, and socio-emotional consequences
Copyright $\odot 2017$ by Thieme Medical Publishers, Inc., 333 Seventh Avenue, New York, NY 10001, USA. Tel: +1(212) 584-4662.
License terms

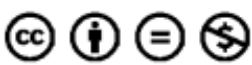


that contribute to prolonged functional impairment following mild BI is key to developing effective treatment practices.

Multiple definitions of mild BI have appeared in extant literature. For the purpose of the present investigation, our interest was in acquired BIs (i.e., injuries to the brain that occur after birth and are "not hereditary, congenital, degenerative, or induced by birth trauma") ${ }^{1}$ that met the severity criteria for clinical identification as mild (i.e., injuries resulting in "confusion or disorientation, loss of consciousness for 30 minutes or less, post-traumatic amnesia for less than 24 hours, and/or other transient neurological abnormalities such as focal signs, seizure, and intracranial lesion not requiring surgery"2 [p. 115]).

Most people sustaining mild BIs recover within a period of a few days or weeks; however, a subset experiences challenges that endure for months or years and interfere with the resumption of pre-injury activities. ${ }^{2-10}$ Researchers have differed in their findings regarding the size of this subset, with some suggesting that as small as $2 \%$ experience chronic challenges, ${ }^{3}$ and others suggesting that as large as $45 \%$ show this type of recovery pattern. ${ }^{11}$ Regardless, the result is that a group of individuals experience long-lasting cognitive, physiological, and/or socio-emotional challenges affecting educational, social, and vocational endeavors following mild BI and who may benefit from receiving accommodations and professional services. $^{12}$

The occurrence of incidents potentially causing mild BI is common in the general population. According to epidemiology reports, $\sim 1.4$ million United States citizens receive emergency room or some other form of medical treatment for mild BIs each year. ${ }^{13}$ Given that these figures include only those individuals who seek medical care, the actual occurrence of mild $\mathrm{BI}$ is likely even higher. In fact, questioning parents in a Midwestern state revealed that $42.11 \%$ of their offspring had sustained one or more substantial blows to the head-some of which were medically documented and some of which were not-before completing high school. ${ }^{14}$ In another study in which parents of elementary school-aged children responded to a short questionnaire, 33.82\% reported that their children had experienced possible BI events. ${ }^{15}$ These statistics confirm that experiencing possible mild BI events before reaching adulthood is relatively commonplace. What remains unclear is the chronic symptomatology and long-term outcome associated with sustaining such injuries.

The uniqueness of every mild BI in terms of mechanism, location, and extent of neurological damage contributes to the wide spectrum of characteristics and challenges displayed by survivors. In general, complaints comprise three main categories: cognitive issues, physiological or somatic issues, and socio-emotional or affective issues. Cognitive complaints include, but are not limited to, deficits in memory, concentration, attention, and organization that affect the speed and quality of information processing and new learning; physiological complaints include headaches, fatigue or decreased energy, blurred vision, sleep disturbances, dizziness or balance problems, sensitivity to light or sound, and tinnitus; and socioemotional complaints include depression, anxiety, panic attacks, and irritability or anger that contribute to social dysfunction. 6,16,17 When one or more of these symptoms persist for an extended period post-injury, an individual may struggle to perform daily activities at a level commensurate with premorbid abilities.

Predicting individuals likely to experience long-term consequences following mild BI has proven difficult. ${ }^{4}$ Given that some researchers have found that females are more susceptible than males to sustaining sports-related concussions, ${ }^{18}$ gender has emerged as a possible demographic factor affecting outcome. ${ }^{7}$ However, conflicting data exist regarding the outcome patterns in that some researchers report no difference between males and females in recovery, ${ }^{10,19,20}$ while other researchers have found that males have a recovery advantage over females. ${ }^{18,21-25}$ Further complicating the use of gender as an outcome predictor is the fact that females tend to be more forthright than males about reporting injuries and seeking medical advice about health complaints in general. ${ }^{26}$ This reporting bias may be prominent regarding mild BIs given the heavy reliance placed on the self-report of incidents and symptoms. The result is that using gender as a long-term prognostic indicator has multiple inherent challenges.

Another demographic factor that may relate to long-term outcome is age at the time of injury. Following severe BI in adults, increasing age corresponds with more unfavorable outcome; ${ }^{27,28}$ among children, a different scenario exists in that those sustaining severe injuries in preschool years tend to fair worse than their older counterparts. ${ }^{29,30}$ An alternate pattern may appear between children and adolescents versus adults following mild BIs because biomechanical, pathophysiological, and neurobehavioral differences make youngsters more susceptible to diffuse neurological damage. ${ }^{31}$ Hence, children and adolescents sustaining mild BIs may experience more extended periods of symptom development and persistence contributing to cognitive, physiological, and psychosocial problems than those experienced by adults. ${ }^{32,33}$ Different recovery patterns from mild BI have yet to be documented between children and adolescents of various ages, ${ }^{29}$ but this remains an area in which additional research is needed. ${ }^{30}$

Researchers have also examined factors such as the severity of initial injury and the appearance of specific symptoms during acute recovery for their prognostic value regarding long-term outcome. Neither has provided a means of predicting who is likely to struggle with enduring impairments following mild $\mathrm{BI},{ }^{6,7}$ although people with multiple complaints during early months post-injury coupled with relatively long periods of post-traumatic amnesia tend to experience worse long-term outcomes than those with few initial complaints and short periods of post-traumatic amnesia do. ${ }^{10}$ However, further complicating the scenario is the tendency for medical professionals to minimize the potential for negative outcomes following mild injuries in an effort to alleviate undue concern-a practice that weakens the likelihood of people linking past events with persistent symptoms. ${ }^{6,34,35}$ Given the difficulty of predicting long-term outcome and the inappropriateness of assuming that all people with mild BIs will experience lasting problems, the intent of this study was to explore among young adults in a post-secondary educational setting a means of identifying 
those with substantive versus negligible persistent symptomatology. Specifically, our purpose was two-fold: (a) to use latent class analysis (LCA) (e.g., diagnostic model) to identify a subset of college students who present a pattern of chronic symptomatology consistent with a diagnosis of mild BI and (b) to use pseudo-class mean equality tests to examine relations between latent classes (i.e., subsets of students who present different patterns of symptomatology following mild BI) and other variables such as BI event characteristics (e.g., loss of consciousness) and academic outcomes. We obtained written approval from the local institutional review board ensuring implementation of adequate protections of human subjects.

\section{Method}

\section{Participants}

The 118 study participants were a subset of 423 predominantly female young adults (female:male $=5.82: 1$ ) who completed surveys contributing to a database about BI in undergraduate college students. All participants provided consent for participation before responding to any survey items. The 118 college students self-reported histories of documented or undocumented mild BIs. The sample was relatively homogenous regarding demographics-that is, participants were primarily Caucasian (94.92\%) female (77.97\%) students who were fairly evenly distributed across freshman (16.95\%), sophomore (30.51\%), junior (26.27\%), and senior $(26.27 \%)$ years in college. Six students reported receiving academic accommodations (e.g., priority registration, extended test time, etc.) at the time of the study.

The 118 participants reported a total of 234 possible BI events (range: $1-8$, median $=1.98$, and standard deviation $[S D]=1.40)$. Sport-related injuries accounted for $49.15 \%$ of the reported events, with traffic accidents and falls being the cause of most of the remaining incidents (i.e., $24.36 \%$ and $12.82 \%$, respectively). Explosions, assaults, abuse, seizures, and other medical events collectively accounted for the remaining $13.67 \%$ of possible BI events. Thirty-one (26.27\%) respondents experienced a loss of consciousness, with seven of those individuals losing consciousness in association with more than one BI event. All but two respondents claimed to have lost consciousness for $<5$ minutes. Both people with longer unconsciousness reported it lasting between 10 and 30 minutes.

\section{Materials and Procedures}

Respondents completed a web-based survey developed and distributed via Qualtrics ${ }^{\odot}$. We developed the survey using conditional logic such that the number and type of questions answered was contingent on previous responses. All participants responded to a minimum of 32 questions; the maximum number of potential questions posed was 1,192 . On average, respondents completed the survey in 12 minutes and 7 seconds (mean $[M]=726.53$ seconds, $S D=422.92$ ).

Survey items related to four topics: daily habits and routines, academic performance, general health, and self-reported potential BI events. Thirty-seven questions were about daily habits (i.e., sleep, nutrition, drug/alcohol use, and exercise routines); academic performance (i.e., grade point average and number of repeated or failed courses over a respondent's academic career); and current health (i.e., 24 cognitive, physiological, and socio-emotional health complaints). We included the 24 symptoms listed in -Table 1 on the survey instrument to query about general health because of previous research establishing these complaints as ones people who have sustained BIs frequently endorse $e^{6,16,17,35-38}$ and because many of the complaints are also endorsed by people who have general health issues not relating to $\mathrm{BI}$.

All remaining survey items used conditional logic to query respondents about potential BI events experienced across their lifespans. We selected BI event categories because of the frequency with which they reportedly cause brain damage according to the Centers for Disease Control and Prevention. ${ }^{39}$ BI categories included being in or near explosions; experiencing whiplash injuries or a blows to the head in traffic accidents, falls, sporting events, assaults, or abuse; seizures, brain infections, strokes, or other medical conditions; and/or loss of consciousness because of near drowning, suffocation, or exposure to toxins. We chose not to use the terms brain injury and concussion within questions to prevent biasing participant responses. Within each category,

Table 1 Physiological, cognitive, and socio-emotional complaints associated with BI and included on the self-report measure

\begin{tabular}{|l|l|l|}
\hline Physiological complaints & Cognitive complaints & Socio-emotional complaints \\
\hline Trouble sleeping & Memory problems & Irritability/mood swings \\
\hline Dizziness & Planning/organization problems & Depression \\
\hline Headaches & Slow thinking/speed of processing & Apathy \\
\hline Light/sound sensitivity & Difficulty learning new information & Relationship difficulties \\
\hline Problems focusing eyes & Attention problems & Flashbacks \\
\hline Balance/coordination problems & Concentration problems & Panic attacks \\
\hline Reduced mobility & Difficulty multitasking & \\
\hline Excessive fatigue & Problems starting or finishing tasks & \\
\hline Pain & Difficulty solving problems & \\
\hline
\end{tabular}

Abbreviation: BI, brain injury. 
respondents could report up to four separate events. If respondents had more than four events to report in a given category, we instructed them to describe their most severe remaining incident as the fourth event. When reporting a potential BI event, respondents provided their age at the time of injury, a short description, symptoms experienced, and the duration of experienced symptoms.

\section{Variables}

Twenty-four BI sequelae grouped into cognitive, physiological, or socio-emotional categories ( - Table 1 ) served to identify symptomatology profiles in an LCA. Each symptom was coded as a dichotomy; hence, the symptom was reported either as present or absent. Three academic outcomes-grade point average, number of failed classes, and number of repeated classes-also served as dependent variables as well as the number of possible BI events a person had experienced and whether a concomitant loss of consciousness occurred.

\section{Data Analysis}

We used Mplus v7.11 to fit a mixture model (i.e., LCA) ${ }^{40}$ to the symptomatology data (e.g., headaches, attention problems, and slow thinking). LCA provides a means for identifying latent (i.e., unobserved) groups-called classes-by finding different patterns or profiles of symptoms among participants. LCA models are becoming more widely used in behavioral science and medicine to explore unobserved heterogeneity within a population and as a statistical method for identifying typologies of individuals or cases. ${ }^{41,42}$

\section{Class Enumeration}

Two-class and three-class models were fit to the symptom data. A two-class model assumes two underlying groups in the population: (1) individuals who recover quickly from a BI event and show little to no symptomatology and (2) individuals who experience persistent symptomatology associated with BI. The three-class model assumes three underlying groups in the population: (1) individuals who recover from the BI with negligible symptomatology; (2) individuals who develop moderate symptomatology across the cognitive, physiological, and socio-emotional categories (e.g., concentration problems, headaches, balance, problems sleeping, and anger control); and (3) individuals who develop high symptomatology across the three symptom categories with particularly clear differentiation in the cognitive domain (e. g., slow thinking, troubling learning new information, and difficulty solving problems). We did not test alternative models with more classes because of the theoretical and practical justification for the three-class model.

\section{Model Fit}

Goodness-of-fit to the model and classification accuracy (i.e., entropy) provided a means of assessing the tenability and usefulness of the three-class model. We used the chi-square likelihood ratio test (LRT) and the bootstrapped likelihood ratio test (BLRT) ${ }^{43}$ to assess model fit. Non-significant chisquare statistics indicate close fit and, thus, the tenability of assuming individuals who sustain BIs comprises three groups. The BLRT compares model fit improvement as classes are added to the model (e.g., a three-class model compared with a two-class model). A significant BLRT test indicates that the model with $k$ classes represents a statistically significant improvement over a model with $k-1$ classes.

\section{Classification Accuracy}

The focus of person-centered analyses, such as LCA, is on determining group membership for each individual in the sample based on a set of observed indicators. Because we are predicting rather than observing group membership, we can express the uncertainty of the prediction as classification accuracy as measured by the entropy statistic. ${ }^{44}$ Entropy values range from 0.00 (completely inaccurate) to 1.00 (completely accurate), with high entropy values $(>0.80)^{45}$ indicating greater confidence in group classification. Models with high entropy are preferable, but entropy does not reveal how well the model represents the data.

\section{Mean Equality Tests}

We specified the model using the AUXILIARY feature in Mplus to allow for testing mean differences in manifest variables, such as academic outcomes across latent classes. This approach uses so-called pseudo-class draws $s^{45,46}$ to account for the uncertainty of class membership by incorporating the posterior probabilities of being in each latent class. The mean equality tests result in a chi-square statistic with one degree of freedom. We computed Cohen's $d$ effect size estimates for significant null hypothesis tests.

\section{Results}

\section{Latent Class Analysis}

\section{Two-Class Model}

The two-class latent model fit the data well as indicated by the non-significant chi-square LRT $\left(\chi^{2}{ }_{(8388519)}=219.33\right.$, $p=1.00)^{\mathrm{a}}$. The non-significant chi-square value suggests that variation in symptomatology following BI can be sufficiently explained by the presence of two latent groups. This model predicted that $22(18.64 \%)$ of the 118 respondents with histories of possible BI were in the persistent symptomatology class, while the other 96 were in the negligible symptomatology class. The $\operatorname{BLRT}_{(14)}=312.83, p<0.001$, also suggested that the sample was not homogenous in symptomatology and that a two-class solution better captures the variation in symptomatology than a one-class solution does (i.e., assuming a homogeneous population). Entropy for this model was 0.93 indicating excellent classification accuracy.

\section{Three-class Model}

The three-class latent model also fit the data well as indicated by the non-significant chi-square LRT $\left(\chi^{2}{ }_{(16777095)}=177.27\right.$, $p=1.00)^{\mathrm{a}}$. The non-significant chi-square value suggests that variation in symptomatology following $\mathrm{BI}$ events can be

\footnotetext{
a The log-likelihood value was replicated on every one of the 100 final stage optimizations.
} 
sufficiently explained by the presence of three latent groups of individuals-a high symptomatology class, a moderate symptomatology class, and a negligible symptomatology class. The significant BLRT $_{(24)}=115.81, p<0.001$, indicated that the three-class solution better captures the variation in symptomatology than the two-class solution does.

This model predicted that $13(11.01 \%)$ participants were in the high symptomatology class, 58 (49.15\%) participants were in the moderate symptomatology class, and the remaining 47 (39.83\%) participants comprised the negligible symptomatology class. - Fig. 1 shows the symptomatology profiles for each latent class in terms of the conditional probability of experiencing each symptom. Symptoms differentiate latent classes when one class demonstrates probabilities $>0.70$, while the other classes demonstrate probabilities $<0.30 .^{41,47}$ Respondents in the high symptomatology class were highly likely (probability $[\mathrm{Pr}]>0.70$ ) to experience headaches, problems with memory, excessive fatigue, slow thinking, difficulty learning new information, problems with attention, problems with concentration, and problems sleeping. In contrast, respondents in the moderate class were moderately likely ( $\mathrm{Pr}>0.30$ ) to experience headaches, excessive fatigue, problems with concentration, and problems sleeping. Taking these sets of probabilities into consideration, the subset of symptoms that best separates the high and moderate classes was problems with memory (relative risk $(R R)=6.53$ ), slow thinking $(R R=8.05)$, difficulty learning new information $(R R=9.38)$, and problems with attention $(R R=5.32)$. The $R R$ ratio indicates the increased likelihood that a person in the high symptomatology class experienced the symptom compared with a person in the moderate symptomatology class did. For example, there was a $553 \%$ increase in the likelihood of a respondent in the high symptomatology class experiencing problems with memory than a respondent in the moderate symptomatology class.

Entropy for the three-class model was 0.92 , thus indicating near perfect classification into the three groups. ${ }^{45}$ In other words, the statistical model clearly differentiated between three patterns of symptomatology following selfreported BI events. Only a handful of respondents ( 8\%) exhibited patterns of symptomatology inconsistent with one of the three groups (i.e., misclassification).

\section{Latent Class Relation to Other Variables}

We used mean equality tests to assess the associations between latent class membership and other variables for the three-class model. Specially, we looked for differences relating to the number of possible $\mathrm{BI}$ events reported, loss of consciousness, grade point average (GPA), number of failed courses, and the number of repeated courses. Regarding BI event characteristics, we hypothesized that individuals in the high symptomatology class would report having experienced a greater number of possible BI events than respondents in the other two latent classes but that no difference would emerge in the loss of consciousness. Regarding academic outcomes, we hypothesized that individuals in the high symptomatology class would demonstrate lower GPAs and higher rates of failed and repeated courses than respondents in the other two latent classes.

The analysis indicated that the number of self-reported BI events did not differ significantly across the three latent

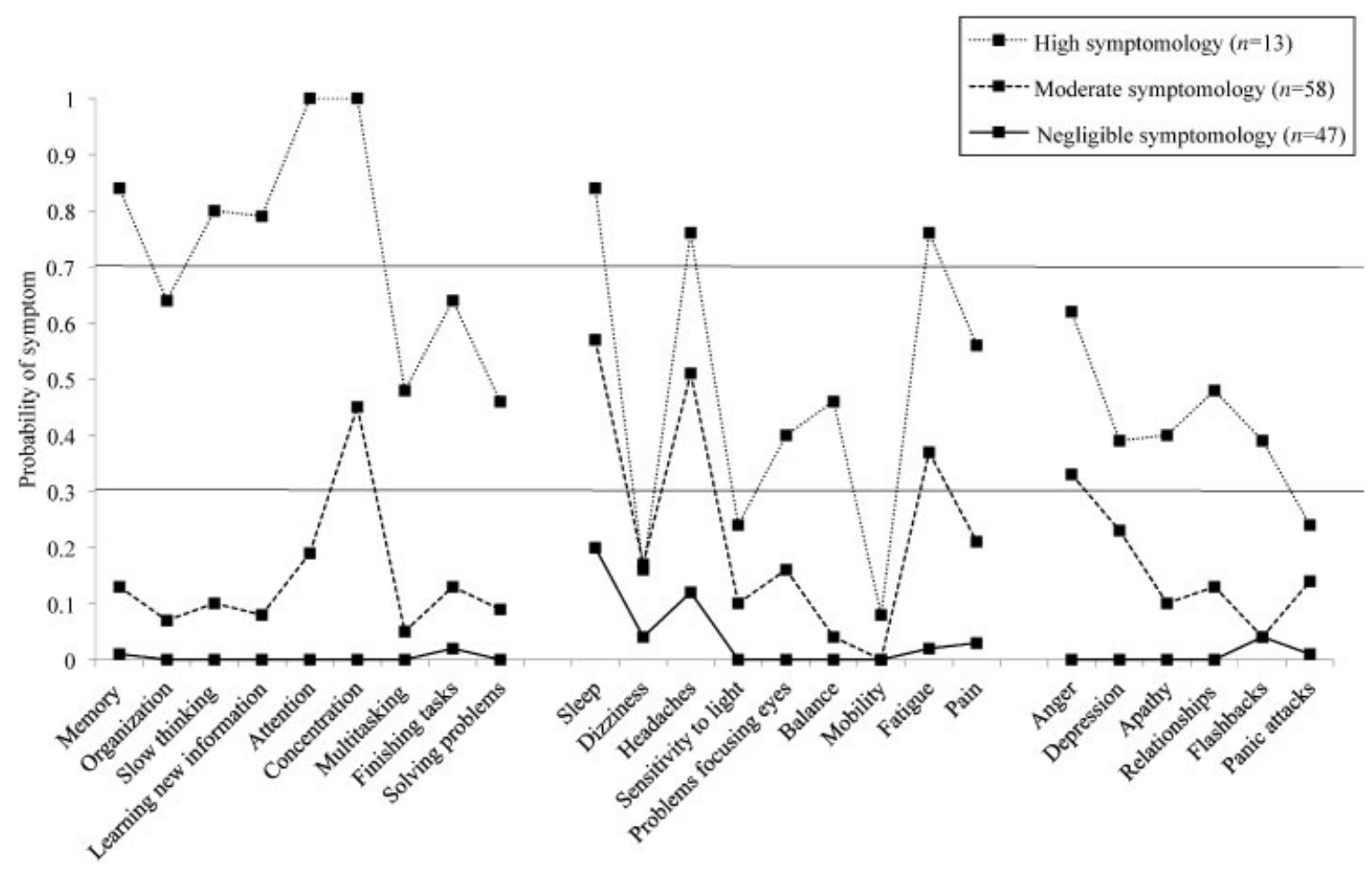

Fig. 1 Symptom probability by latent class for three-class model. 
classes, but that respondents in the high symptomatology class were less likely to experience a loss of consciousness compared with those in the moderate symptomatology class, $\mathrm{X}^{2}(1)=6.02, p<0.05$. People in the high symptomatology class had statistically lower GPA $(M=3.15$, SD $=0.41)$ compared with those in the moderate symptomatology class $(M=3.46, S D=0.40), \mathrm{X}^{2}{ }_{(1)}=6.11, p<0.05, d=-0.78$, and marginally lower GPA compared with those in the negligible symptomatology class $(M=3.41, \mathrm{SD}=0.52), \mathrm{X}_{(1)}^{2}=3.58$, $p=0.059, d=-0.55$. Respondents in the high symptomatology class also failed more courses compared with those both in the moderate $\left(X_{(1)}^{2}=4.31, p<0.05, d=0.99\right)$ and negligible classes $\left(X^{2}{ }_{(1)}=3.94, p<0.05, d=0.86\right)$. Individuals in the high symptomatology class repeated more courses than those reported by the respondents in the moderate class and negligible class, but the differences were not statistically significant at the $0.05 \alpha$-level. No statistically significant differences emerged between respondents in the moderate symptomatology class and those in the negligible symptomatology class for any of the three academic dependent variables.

\section{Discussion}

The primary purpose of this study was to determine whether latent classes exist with regard to the cognitive, physiological, and socio-emotional symptomatology experienced as chronic health complaints by young adults who self-identify as having sustained one or more mild BIs in their lifetime. The obtained three-class model predicts that $11 \%$ of people with self-reported mild BIs will experience high symptomatology as chronic health issues, $49 \%$ will experience moderate chronic symptomatology, and $40 \%$ will experience negligible chronic symptomatology. Furthermore, many of the symptoms distinguishing the high symptomatology class from the other two classes are cognitive in nature. Specifically, people in the high symptomatology class have a high probability of reporting and are more than five times more likely to complain of problems with memory, speed of processing, learning new information, and paying attention than people in the moderate or negligible symptomatology classes. Although endorsed with only moderate frequency by people with high symptomatology, problems with organization, multitasking, focusing the eyes, balancing, and having flashbacks are also more than five times more likely than these in the moderate or negligible symptomatology classes. The magnitude of probability differences in the occurrence of these symptoms validates the notion that people who endorse them comprise a distinct group from those who do not. Of note, however, is the finding that socio-emotional symptomatology, in general, did not emerge as a differentiating factor and was not endorsed by a high percentage of respondents in any of the latent classes. This is somewhat surprising given that depression, anxiety, and problems with coping are reportedly frequent complaints following mild BI. ${ }^{12,37,38}$

Another purpose of this study was to assess associations between latent class membership and various BI event characteristics. One of the BI event characteristics evaluated was loss of consciousness during a BI event. Given extant research suggesting loss of consciousness does not serve as a good predictor of mild BI outcome, ${ }^{48,49}$ we did not expect this variable to emerge as a distinguishing feature among any of the latent classes. Performance of the analysis revealed that our hypothesis was inaccurate; however, it was so in an unexpected way. Specifically, our finding was that those respondents classified with high symptomatology were significantly less likely to have lost consciousness than those with moderate symptomatology and did not differ in loss of consciousness likelihood from the negligible symptomatology group. Hence, although different from our original hypothesis, the finding supports the idea that loss of consciousness following mild BI is not predictive of chronic symptoms.

A second characteristic evaluated with regard to latent class distinctions was the total number of self-reported BI events a person sustained. We expected multiple BI events would increase the likelihood of a person experiencing chronic cognitive, physiological, or socio-emotional complaints, because recent literature has linked chronic traumatic encephalopathy (CTE) with repeated concussions and mild BIs sustained across the lifespan. ${ }^{12}$ However, the expected association between number of BI events and latent class membership did not appear from the data analysis. Instead, respondents with single mild BI events were equally likely as those reporting multiple events to have chronic complaints that led to their membership in the high symptomatology class. This finding may reflect the fact that our participants, as a whole, did not report the large numbers of BI events-such as are sustained by athletes, people with occupations that repeatedly expose them to explosions or subconcussive impacts, or people with histories of seizures or head-banging episodes-typically associated with CTE. ${ }^{50,51}$ Also, given that our participant sample was comprised of students currently pursuing undergraduate college degrees, sufficient time was unlikely to have yet elapsed for CTE symptoms to appear. Still, the finding of no association between the number of possible BI events and latent class membership is important, because it confirms that substantial chronic effects can follow a single concussion/mild BI, and chronic symptomatology following single events may be comparable to that experienced by people sustaining repeated injuries.

A final purpose of this study was to assess associations between latent class membership and academic outcomes. Regarding GPA and number of failed classes, the obtained results were consistent with our initial hypotheses that those in the high symptomatology class would experience greater challenges academically than those experienced by their peers in the moderate and negligible symptomatology classes. These findings are also consistent with those of other researchers who have examined the academic performance of college students with histories of BIs. ${ }^{16,17,52}$ In particular, the cognitive challenges experienced by individuals with BIs appear to have a negative effect on the mastery of academic material, thus necessitating greater studying time, effort, and 
application of supplemental learning strategies than these needed by students without BIs. Despite these struggles, however, students with BI histories seldom seek support services available with post-secondary institutions. ${ }^{16,17}$ More extensive investigation of academic performance and struggles than is possible from examining overall GPA or the number of failed classes may validate the obtained findings and may identify factors likely to support the academic pursuits of young adults with BIs.

\section{Clinical Implications}

The near perfect classification of study participants into three latent classes regarding BI symptomatology suggests that using a questionnaire, such as the one developed for this research, and applying the established class distinction criteria may serve as a means of identifying young adults with persistent challenges secondary to mild BI. This is important because the findings suggest that $\sim 11 \%$ of college students experience high symptomatology on a persistent basis. Identifying individuals with high symptomatology and providing them with appropriate treatments, supports, and accommodations is important for promoting their success in future academic, social, and vocational endeavors, especially given that many of the reported symptoms are cognitive in nature. Furthermore, because treatments appropriate for individuals with symptomatology resulting from BI may differ from those appropriate for people with similar complaints, but stemming from other etiologies, ${ }^{53}$ correct identification of the underlying source of an individual's chronic complaints is critical.

Many professionals, as well as members of the lay public, have long assumed that the effects of mild BI are transient; however, evidence is mounting that individuals may experience long-term post-concussive symptoms for months, years, or even decades following injury. ${ }^{3-10}$ Identifying long-term effects of injury and those individuals who are experiencing chronic symptoms is likely to enhance treatment relating to academic, social, and vocational outcomes. This is of particular importance when injuries occur to children, adolescents, or young adults whose brains are still undergoing developmental changes and who may be most susceptible to negative consequences associated with diffuse neurological damage. ${ }^{31-33}$

\section{Limitations}

Several limitations regarding the sample population used to perform the LCA are worthy of note. First, the sample population was limited to individuals pursuing undergraduate degrees. Although targeting this population was our intent, the consequent age restriction regarding data collection may limit generalization of the findings. Second, the sample was disproportionately female and may, consequently, not adequately reflect the symptomatology experienced by males who have experienced incidents of mild BI. Third, the sample size was relatively small for performing an LCA, and the model included only dichotomous indicators of symptomatology. Replication and extension of the research with larger samples and using other indicators, such as symptom severity and symptom duration, may prove advantageous.

\section{Conclusions}

The findings of this study suggest that within the subset of college students who report having experienced one or more possible BI events across their lifespans, specific problems are paramount in distinguishing those displaying chronic high symptomatology from their peers with moderate and negligible symptomatology. The most salient of these symptoms relate to cognitive processes and include memory challenges, slowed speed of processing, difficulty learning new information, and problems paying attention. The observed magnitude of probability differences across the identified latent classes validates the notion that people endorsing these symptoms as chronic health challenges comprise a distinct group from others who have also experienced possible mild BIs. Furthermore, the fact that high symptomatology class individuals differed academically from their counterparts displaying fewer chronic symptoms suggests a functional consequence of mild BI for this subset of college students. Taken as a whole, these results suggest that about 1 in 10 undergraduate students who self-report a history of one or more possible BI events experiences chronic symptomatology affecting physiological, socio-emotional, and cognitive status. Because these individuals may benefit from physical health-, mental health-, and/or academicrelated services, identifying them prior to or during their attendance at college may be advantageous.

\section{Conflict of Interests}

The authors have no conflicts of interest to declare.

\section{References}

1 Brain Injury Association of America. What is the difference between an acquired brain injury and a traumatic brain injury? 2015. Available at: www.biausa.org/FAQRetrieve.aspx?ID $=43913$

2 Carroll LJ, Cassidy JD, Holm L, Kraus J, Coronado VG; WHO Collaborating Centre Task Force on Mild Traumatic Brain Injury. Methodological issues and research recommendations for mild traumatic brain injury: the WHO Collaborating Centre Task Force on Mild Traumatic Brain Injury. J Rehabil Med 2004;43(43, Suppl):113-125

3 Barlow KM, Crawford S, Stevenson A, Sandhu SS, Belanger F, Dewey D. Epidemiology of postconcussion syndrome in pediatric mild traumatic brain injury. Pediatrics 2010;126(02):e374-e381

4 Carroll LJ, Cassidy JD, Peloso PM, et al; WHO Collaborating Centre Task Force on Mild Traumatic Brain Injury. Prognosis for mild traumatic brain injury: results of the WHO Collaborating Centre Task Force on Mild Traumatic Brain Injury. J Rehabil Med 2004;36 (43, Suppl):84-105

5 Emanuelson I, Andersson Holmkvist E, Björklund R, Stålhammar D. Quality of life and post-concussion symptoms in adults after mild traumatic brain injury: a population-based study in western Sweden. Acta Neurol Scand 2003;108(05):332-338

6 McAllister TW, Arciniegas D. Evaluation and treatment of postconcussive symptoms. NeuroRehabilitation 2002;17(04):265-283

7 McCrory P, Meeuwisse WH, Aubry M, et al. Consensus statement on concussion in sport: the 4th International Conference on Concussion in Sport held in Zurich, November 2012. Br J Sports Med 2013;47(05):250-258 
8 Røe C, Sveen U, Alvsåker K, Bautz-Holter E. Post-concussion symptoms after mild traumatic brain injury: influence of demographic factors and injury severity in a 1-year cohort study. Disabil Rehabil 2009;31(15):1235-1243

9 Sterr A, Herron KA, Hayward C, Montaldi D. Are mild head injuries as mild as we think? Neurobehavioral concomitants of chronic post-concussion syndrome. BMC Neurol 2006;6:7

10 van der Naalt J, van Zomeren AH, Sluiter WJ, Minderhoud JM. One year outcome in mild to moderate head injury: the predictive value of acute injury characteristics related to complaints and return to work. J Neurol Neurosurg Psychiatry 1999;66(02):207-213

11 Stålnacke BM, Björnstig U, Karlsson K, Sojka P. One-year follow-up of mild traumatic brain injury: post-concussion symptoms, disabilities and life satisfaction in relation to serum levels of S-100B and neurone-specific enolase in acute phase. J Rehabil Med 2005; 37(05):300-305

12 Daneshvar DH, Riley DO, Nowinski CJ, McKee AC, Stern RA, Cantu RC. Long-term consequences: effects on normal development profile after concussion. Phys Med Rehabil Clin N Am 2011;22(04): 683-700, ix

13 Bazarian JJ, McClung J, Shah MN, Cheng YT, Flesher W, Kraus J. Mild traumatic brain injury in the United States, 1998-2000. Brain Inj 2005;19(02):85-91

14 Hux K, Bond V, Skinner S, Belau D, Sanger D. Parental report of occurrences and consequences of traumatic brain injury among delinquent and non-delinquent youth. Brain Inj 1998;12(08):667-681

15 Hux K, Dymacek R, Childers C. Possible brain injury events and symptoms in elementary school children. Brain Inj 2013;27(12): 1348-1355

16 Kennedy MR, Krause MO, Turkstra LS. An electronic survey about college experiences after traumatic brain injury. NeuroRehabilitation 2008;23(06):511-520

17 Krause M, Richards S. Prevalence of traumatic brain injury and access to services in an undergraduate population: a pilot study. Brain Inj 2014;28(10):1301-1310

18 Dick RW. Is there a gender difference in concussion incidence and outcomes? Br J Sports Med 2009;43(Suppl 1):i46-i50

19 Moore DW, Ashman TA, Cantor JB, Krinick RJ, Spielman LA. Does gender influence cognitive outcome after traumatic brain injury? Neuropsychol Rehabil 2010;20(03):340-354

20 Ponsford J, Draper K, Schönberger M. Functional outcome 10 years after traumatic brain injury: its relationship with demographic, injury severity, and cognitive and emotional status. J Int Neuropsychol Soc 2008;14(02):233-242

21 Broshek DK, Kaushik T, Freeman JR, Erlanger D, Webbe F, Barth JT. Sex differences in outcome following sports-related concussion. J Neurosurg 2005;102(05):856-863

22 Covassin T, Schatz P, Swanik CB. Sex differences in neuropsychological function and post-concussion symptoms of concussed collegiate athletes. Neurosurgery 2007;61(02):345-350, discussion 350-351

23 Farace E, Alves WM. Do women fare worse: a metaanalysis of gender differences in traumatic brain injury outcome. J Neurosurg 2000;93(04):539-545

24 Santa Maria MP, Pinkston JB, Miller SR, Gouvier WD. Stability of postconcussion symptomatology differs between high and low responders and by gender but not by mild head injury status. Arch Clin Neuropsychol 2001;16(02):133-140

25 Kraus JF, Peek-Asa C, McArthur D. The independent effect of gender on outcomes following traumatic brain injury: a preliminary investigation. Neurosurg Focus 2000;8(01):e5

26 Galdas PM, Cheater F, Marshall P. Men and health help-seeking behaviour: literature review. J Adv Nurs 2005;49(06):616-623

27 Hukkelhoven CW, Steyerberg EW, Rampen AJ, et al. Patient age and outcome following severe traumatic brain injury: an analysis of 5600 patients. J Neurosurg 2003;99(04):666-673

28 Steyerberg EW, Mushkudiani N, Perel P, et al. Predicting outcome after traumatic brain injury: development and international validation of prognostic scores based on admission characteristics. PLoS Med 2008;5(08):e165, discussion e165

29 Anderson V, Catroppa C, Morse S, Haritou F, Rosenfeld J. Functional plasticity or vulnerability after early brain injury? Pediatrics 2005;116(06):1374-1382

30 Babikian T, Asarnow R. Neurocognitive outcomes and recovery after pediatric TBI: meta-analytic review of the literature. Neuropsychology 2009;23(03):283-296

31 Kirkwood MW, Yeates KO, Wilson PE. Pediatric sport-related concussion: a review of the clinical management of an oftneglected population. Pediatrics 2006;117(04):1359-1371

32 Huh JW, Widing AG, Raghupathi R. Midline brain injury in the immature rat induces sustained cognitive deficits, bihemispheric axonal injury and neurodegeneration. Exp Neurol 2008;213(01): 84-92

33 Pullela R, Raber J, Pfankuch T, et al. Traumatic injury to the immature brain results in progressive neuronal loss, hyperactivity and delayed cognitive impairments. Dev Neurosci 2006;28(4-5):396-409

34 Cantor JB, Gordon WA, Schwartz ME, Charatz HJ, Ashman TA, Abramowitz $S$. Child and parent responses to a brain injury screening questionnaire. Arch Phys Med Rehabil 2004;85(04, Suppl 2):S54-S60

35 Gordon WA, Brown M, Sliwinski M, et al. The enigma of "hidden" traumatic brain injury. J Head Trauma Rehabil 1998;13(06): 39-56

36 Gordon WA, Haddad L, Brown M, Hibbard MR, Sliwinski M. The sensitivity and specificity of self-reported symptoms in individuals with traumatic brain injury. Brain Inj 2000;14(01):21-33

37 Panayiotou A, Jackson M, Crowe SF. A meta-analytic review of the emotional symptoms associated with mild traumatic brain injury. J Clin Exp Neuropsychol 2010;32(05):463-473

38 Whittaker R, Kemp S, House A. Illness perceptions and outcome in mild head injury: a longitudinal study. J Neurol Neurosurg Psychiatry 2007;78(06):644-646

39 Faul M, Xu L, Wald MM, Coronado VG. Traumatic Brain Injury in the United States: Emergency Department Visits, Hospitalizations, and Deaths 2002-2006. Atlanta, GA: Centers for Disease Control and Prevention National Center for Injury Prevention and Control; 2010

40 Lazarsfeld PF, Henry NW. Latent Structure Analysis. Boston, MA: Houghton Mifflin Co.; 1968

41 Muthén B. Latent variable analysis. In: Kaplan D, ed. The Sage Handbook of Quantitative Methodology for the Social Sciences. Thousand Oaks, CA: Sage Publications; 2004:345-368

42 Rasmussen ER, Neuman RJ, Heath AC, Levy F, Hay DA, Todd RD. Replication of the latent class structure of Attention-Deficit/ Hyperactivity Disorder (ADHD) subtypes in a sample of Australian twins. J Child Psychol Psychiatry 2002;43(08):1018-1028

43 McLachlan G, Peel D. Finite Mixture Models. New York: Wiley; 2000

44 Celeux G, Soromenho G. An entropy criterion for assessing the number of clusters in a mixture model. J Classif 1996;13(02): 195-212

45 Clark SL, Muthén B. Relating latent class analysis results to variables not included in the analysis. 2009. Available at: www. statmodel.com/download/relatinglca.pdf

46 Asparouhouv T, Muthen B. Wald test of mean equality for potential latent class predictors in mixture modeling. 2007. Available at: http://www.statmodel.com/download/MeanTest1.pdf

47 Muthén B. Should substance use disorders be considered as categorical or dimensional? Addiction 2006;101(Suppl 1):6-16

48 Collins MW, Iverson GL, Lovell MR, McKeag DB, Norwig J, Maroon J. On-field predictors of neuropsychological and symptom deficit following sports-related concussion. Clin J Sport Med 2003; 13(04):222-229

49 Lovell MR, Iverson GL, Collins MW, McKeag D, Maroon JC. Does loss of consciousness predict neuropsychological decrements after concussion? Clin J Sport Med 1999;9(04):193-198 
e22 Brain Injury Symptomatology Hux et al.

50 Gavett BE, Stern RA, Cantu RC, Nowinski CJ, McKee AC. Mild traumatic brain injury: a risk factor for neurodegeneration. Alzheimers Res Ther 2010;2(03):18-21

51 McKee AC, Cantu RC, Nowinski CJ, et al. Chronic traumatic encephalopathy in athletes: progressive tauopathy after repetitive head injury. J Neuropathol Exp Neurol 2009;68(07): 709-735
52 Hux K, Bush E, Zickefoose S, Holmberg M, Henderson A, Simanek G. Exploring the study skills and accommodations used by college student survivors of traumatic brain injury. Brain Inj 2010;24(01):13-26

53 Ylvisaker M, Turkstra LS, Coelho C. Behavioral and social interventions for individuals with traumatic brain injury: a summary of the research with clinical implications. Semin Speech Lang 2005;26(04):256-267 